\title{
Hibiscus sabdariffa Mucilage as a Disintegrant in Formulating Fast Dissolving Tablets
}

\author{
S. B. Shirsand ${ }^{1}$, Shivanand ${ }^{1}$, Shailashri ${ }^{1}$, G. G. Keshavshetti ${ }^{2}$ and V. J onathan ${ }^{1}$ \\ ${ }^{1}$ Department of Pharmaceutical Technology, H.K.E. Society’s College of Pharmacy, \\ Gulbarga-585105 (Karnataka), India \\ ${ }^{2}$ Department of Pharmaceutics, S.V.E. Trust's College of Pharmacy, Humnabad-585 330
}

Dist- Bidar (Karnataka), India

(Received: July 09, 2015; Accepted: July 28, 2016; Published (web): December 27, 2016)

\begin{abstract}
The aim of the present work was to prepare and evaluate fast dissolving tablets of nebivolol with a view to enhance patient compliance and minimize the side effects. In this study, fast dissolving tablets of nebivolol were formulated by direct compression method using mucilages of tapioca seeds (Manihot esculenta), basella climb (Basella alba), red sorrel (Hibiscus sabdariffa) as natural disintegrants and crosspovidone as a synthetic superdisintegrant in different ratios with directly compressible mannitol (Pearlitol SD 200) as a diluent to enhance the mouth feel. The prepared formulations were evaluated for hardness, friability, drug content, in vitro dispersion time, wetting time, water absorption ratio, in vitro drug release, stability and excipients interaction. Among all the formulations, the formulation $\left(\mathrm{FHD}_{3}\right)$ containing $8 \% \mathrm{w} / \mathrm{w}$ mucilage of Hibiscus sabdariffa was the overall best formulation ( $\mathrm{t}_{50 \%} 1.7 \mathrm{~min}$ ) based on in vitro drug release studies. Stability studies on the formulations indicated that there are no significant changes in drug content and in vitro dispersion time $(\mathrm{p}<0.05)$. From the above studies, it can be concluded that fast dissolving tablets of nebivolol can be prepared using different mucilages as natural disintegrants for faster dispersion and disintegration in the mouth.
\end{abstract}

Key words: Fast dissolving tablets, nebivolol , crosspovidone, Hibiscus sabdariffa, natural disintegrants

\section{INTRODUCTION}

Recent advances in novel drug delivery system (NDDS) aim to enhance safety and efficacy of drug molecule by formulating a convenient dosage form for administration and to achieve better patient compliance. Modified formulation with such approach is the fast dissolving tablets of nebivolol, an anti-hypertensive drug given orally to reduce blood pressure. ${ }^{1}$ Many patient express difficulties in swallowing tablet and hard gelatin capsules, leading to non-compliance and ineffective therapy. ${ }^{2}$ Other problems experienced in conventional oral dosage forms include patients with mental illness, uncooperative behavior and those suffering from nausea, motion sickness, sudden episodes of allergic

Correspondence to: S.B. Shirsand

Mobile: 98440 79489, 08472-221392

E-mail:shirsand@rediffmail.com

Dhaka Univ. J. Pharm. Sci. 15(2): 143-149, 2016 (December) attack and coughing. ${ }^{3-5}$ Thus, the concept of formulating fast dissolving tablets of nebivolol evolved, which offers a suitable and practical approach in serving the desired objective of faster disintegration and dissolution characteristics with potentially increased bioavailability.

Mucilage is most commonly used as adjuvant in the manufacture of different pharmaceutical dosage form. They possess a variety of pharmaceutical properties, which include binding, disintegrating, suspending, emulsifying and sustaining properties at different proportions in various pharmaceutical dosage forms. The synthetic polymer used as excipients suffer from many disadvantages such as high cost, toxicity, non-biodegradability and environmental pollution during their synthesis. ${ }^{6-9}$ Natural mucilage are preferred over semi-synthetic and synthetic materials, due to their non-toxic, low cost, free availability, emollient and non-irritating 
nature. ${ }^{10,11} H$. sabdariffa contains proteins, amino acids, flavanoids, organic acids and their derivatives. It also contains vitamins $\mathrm{B}_{1}, \mathrm{C}$ and beta-carotine. It is medicinally used as antihypertensive, laxative, purgative, diuretics and in treatment of nervous diseases. Manihot esculenta, Basella alba and Hibiscus sabdariffa mucilage are easily available having low cost and non toxicity as compared to synthetic disintegrants.

In the present study, the fast dissolving tablets of nebivolol were prepared by direct compression method using natural and synthetic disintergrants to compare the efficiency of different natural and synthetic disintegrants.

\section{MATERIALS AND METHODS}

Nebivolol was a gift sample from Cadila Pharma. Ltd., Gujarat. Crospovidone was obtained from Wockhardt Research Centre, Aurangabad, Maharashtra, India. Micro-crystalline cellulose was gift sample from Alkem Labs Pvt. Ltd., Mumbai, Maharashtra, India. All the other chemicals were of analytical grade.

Extraction of Hibiscus sabdariffa mucilage. The fresh samples were cleaned using running tap water and dried in a good air draft under shade overnight until constant weight or in oven at $42^{\circ} \mathrm{C}$. Then the samples were ground into fine particles. The powder was soaked and stirred in 95\% ethanol in water in a ratio of $1: 10(\mathrm{w} / \mathrm{v})$. Then the samples were taken in muslin cloth to separate the crude extract. The crude extract was stored at $4{ }^{0} \mathrm{C}$ which forms solid mass for further use ${ }^{12}$.

Extraction of other natural mucilages. Mucilages from Manihot esculenta and Basella alba were extracted by following the same method described above.

Formulations of nebivolol fast dissolving tablets. Fast dissolving tablets of nebivolol were prepared by direct compression method, using mucilage of M. esculenta, B. alba and H. sabdariffa as natural disintegrants and crosspovidone (CP) as a synthetic superdisintegrant in different ratios and directly compressible mannitol as diluent. All the ingredients were passed through 60 number mesh separately. The drug and mannitol were mixed by small portion of both each time and blending it to get a uniform mixture and kept aside. Then the ingredients were weighed and mixed in geometrical order and tablets were compressed at $7 \mathrm{~mm}$ size to get a tablet of $150 \mathrm{mg}$ weight. ${ }^{13}$ The tablets were prepared according to the formulae shown in table 1.

Evaluation of tablets. The prepared batches of formulation were evaluated for the pre-compression parameters like bulk density, tapped density, angle of repose and Carr's index as shown in table 2 and post compression parameters such as drug content uniformity, weight variation, hardness, friability, thickness, in vitro dispersion time, in vitro drug release and stability studies (Table 3) ${ }^{14,15}$

Weight variation. The weight variation test was done according to the USP where twenty tablets were selected randomly and weighed individually, and the individual weight was compared with average weight for the determination of weight variation.

Tablet hardness. The hardness of each batch of tablet was checked by using digital hardness tester. The hardness was measured in terms of $\mathrm{kg} / \mathrm{cm}^{2}$. Three tablets were chosen randomly and tested for hardness. The average hardness of three determinations was calculated.

Friability. Ten tablets were weighed and the initial weight of these tablets was recorded and placed in Roche friabilator and rotated at the speed of $25 \mathrm{rpm}$ for 100 revolutions. Then tablets were removed from the friabilator, dusted off and again weighed. Percentage friability was calculated by using the formula:

$$
\% \text { Friability }=\frac{\text { Initial weight of the tablets }- \text { Final weight of the tablets }}{\text { Initial weight of the tablets }} \times 100
$$


Tablet thickness. Thickness was measured using Vernier calipers. It was determined by checking the thickness of three tablets from each formulation.

Drug content uniformity. Ten tablets were weighed and powdered, a quantity of powder equivalent to $5 \mathrm{mg}$ of nebivolol was transferred to a $50 \mathrm{ml}$ volumetric flask and dissolved in $40 \mathrm{ml}$ methanol. The drug was extracted into the methanol by vigorously shaking for 15 minutes. Then the volume was adjusted to $50 \mathrm{ml}$ with methanol and the liquid was filtered. The nebivolol content was determined by UV-spectrophotometry by measuring the absorbance at $281 \mathrm{~nm}$ after appropriate dilution with methanol. The drug content was calculated using the standard calibration curve. The mean percent drug content was calculated as an average of three determinations.

Wetting time and water absorption ratio. A piece of tissue paper folded twice was placed in a small petridish containing $6 \mathrm{ml}$ of water. A tablet was put on the paper and the time required for complete wetting was measured. The wet tablet was then weighed (see in Table 3). ${ }^{16}$

The water absorption ratio ' $\mathrm{R}$ ' was determined using following equation:

$$
R=100 \times\{\mathrm{Wa}-\mathrm{Wb} \mid \mathrm{Wb}\}
$$

where,

$\mathrm{W}_{\mathrm{a}}$ = weight of tablet before water absorption,

$\mathrm{W}_{\mathrm{b}}$ = weight of tablet after water absorption.

In vitro dispersion time. Tablet was added to 10 $\mathrm{ml}$ of $\mathrm{pH} 6.8$ phosphate buffer solution at $37 \pm 0.5^{\circ} \mathrm{C}$. Time required for complete dispersion of a tablet was measured (Table 3$)^{17}$.

In vitro dissolution study. In vitro dissolution of nebivolol fast dissolving tablets was studied in USP XXII type-II dissolution apparatus (Electrolab USP TDT-06T) employing a paddle stirrer. $900 \mathrm{ml}$ of $\mathrm{pH}$ 6.8 phosphate buffer was used as dissolution medium. The stirrer was adjusted to rotate at $50 \mathrm{rpm}$. The temperature of dissolution media was previously warmed to $37 \pm 0.5^{\circ} \mathrm{C}$ and was maintained throughout the experiment. One tablet was used in each test, $5 \mathrm{ml}$ of sample of dissolution medium were withdrawn by means of syringe fitted with pre-filter at known intervals of time and analyzed for drug release by measuring the absorbance at $281 \mathrm{~nm}$. The volume withdrawn at each time interval was replaced with fresh quantity of dissolution medium ${ }^{18}$.

Accelerated stability studies. Stability studies on the promising formulation $\left(\mathrm{FHD}_{3}\right)$ were carried out by storing 15 tablets in amber coloured screw capped bottle at elevated temperature of $40 \pm 2{ }^{0} \mathrm{C}$ / 75\% RH (Stability chamber, Oswald) for 3 months. At an interval of one month, the tablets were visually examined for any physical changes, analysed for percent drug content and in vitro dispersion time.

\section{RESULTS AND DISCUSSION}

In the present work fast dissolving tablets of nebivolol were prepared by direct compression method, employing mucilages of Tapioca seeds (Manihot esculenta), Basella climb (Basella alba) and Red sorrel ( $H$. sabdariffa) as natural disintegrants and crosspovidone as a synthetic superdisintegrant in different ratios and mannitol as a diluent. A total of 13 formulations and a control formulation $\mathrm{FC}_{0}$ (without superdisintegrant) were designed. All the blends were free flowing having angle of repose $<30^{\circ}$, Carr's index $<15 \%$, tapped density $<0.640$, bulk density $<0.570$ and hausner's ratio $<1.15$ indicating all the blends have values within the IP limits (given in Table 2).

Tablets obtained were of uniform weight (due to uniform die fill) with acceptable variation as per IP specification i.e. below $\pm 7.5 \%$. Drug content, hardness, water absorption ratio and wetting time were found to be in the range of 97.82 to $98.47 \%, 2.6$ to $2.9 \mathrm{~kg} / \mathrm{cm}^{2}, 68.11 \%$ to $95.45 \%$ and 18.14 to 68.74 sec respectively (given in table 3 ). Friability value of the prepared tablets was found to be less than $1 \%$ (an indication of good mechanical resistance of tablets) .

Among all the designed formulations, the formulation $\mathrm{FHD}_{3}$ (containing 8\% w/w of Hibiscus sabdariffa) was found to be promising. The in vitro dispersion time, wetting time and water absorption 
Table 1. Formulations of nebivolol fast dissolving tablets prepared by direct compression method.

\begin{tabular}{|c|c|c|c|c|c|c|c|c|c|c|c|c|c|}
\hline \multirow{2}{*}{ Ingredients } & \multicolumn{13}{|c|}{ Formulation Code } \\
\hline & $\mathrm{FC}_{0}$ & $\mathrm{FCP}_{1}$ & $\mathrm{FCP}_{2}$ & $\mathrm{FCP}_{3}$ & $\mathrm{FTS}_{1}$ & $\mathrm{FTS}_{2}$ & $\mathrm{FTS}_{3}$ & $\mathrm{FBA}_{1}$ & $\mathrm{FBA}_{2}$ & $\mathrm{FBA}_{3}$ & $\mathrm{FHD}_{1}$ & $\mathrm{FHD}_{2}$ & $\mathrm{FHD}_{3}$ \\
\hline Drug & 5 & 5 & 5 & 5 & 5 & 5 & 5 & 5 & 5 & 5 & 5 & 5 & 5 \\
\hline $\begin{array}{l}\text { Mucilage of } M \text {. } \\
\text { esculenta }\end{array}$ & - & - & - & - & 3 & 6 & 12 & - & - & - & - & - & - \\
\hline Mucilage of B. alba & - & - & - & - & - & - & - & 3 & 6 & 12 & - & - & - \\
\hline $\begin{array}{l}\text { Mucilage of } H \text {. } \\
\text { subdariffa }\end{array}$ & - & - & - & - & - & - & - & - & - & - & 3 & 6 & 12 \\
\hline Crosspovidone & - & 3 & 6 & 12 & - & - & - & - & - & - & - & - & - \\
\hline MCC PH102 & 30 & 30 & 30 & 30 & 30 & 30 & 30 & 30 & 30 & 30 & 30 & 30 & 30 \\
\hline Aspartane & 3 & 3 & 3 & 3 & 3 & 3 & 3 & 3 & 3 & 3 & 3 & 3 & 3 \\
\hline Pineapple Flavour & 3 & 3 & 3 & 3 & 3 & 3 & 3 & 3 & 3 & 3 & 3 & 3 & 3 \\
\hline Talc & 3 & 3 & 3 & 3 & 3 & 3 & 3 & 3 & 3 & 3 & 3 & 3 & 3 \\
\hline SSF ( & 1.5 & 1.5 & 1.5 & 1.5 & 1.5 & 1.5 & 1.5 & 1.5 & 1.5 & 1.5 & 1.5 & 1.5 & 1.5 \\
\hline $\begin{array}{l}\text { Mannitol (Pearlitol } \\
\text { SD200) }\end{array}$ & 104.5 & 101.5 & 98.5 & 92.5 & 101.5 & 98.5 & 92.5 & 101.5 & 98.5 & 92.5 & 101.5 & 98.5 & 92.5 \\
\hline Total weight & 150 & 150 & 150 & 150 & 150 & 150 & 150 & 150 & 150 & 150 & 150 & 150 & 150 \\
\hline
\end{tabular}

FTS- Formulation containing mucilage of Manihot esculenta powder, $\mathrm{FC}_{0^{-}}$(Control) Formulation without super disintegrant , FCPFormulation containing crosspovidone, FHD- Formulation containing mucilage of Hibiscus subdariffa, FBA- Formulation containing mucilage of Basella alba.

Table 2. Pre-compression parameters of formulations prepared by direct compression method.

\begin{tabular}{lccccccccccccc}
\hline \multirow{2}{*}{ Parameters } & \multicolumn{10}{c}{ Formulation code } \\
\cline { 2 - 12 } & $\mathrm{FC}_{0}$ & $\mathrm{FTS}_{1}$ & $\mathrm{FTS}_{2}$ & $\mathrm{FTS}_{3}$ & $\mathrm{FBA}_{1}$ & $\mathrm{FBA}_{2}$ & $\mathrm{FBA}_{3}$ & $\mathrm{FHD}_{1}$ & $\mathrm{FHD}_{2}$ & $\mathrm{FHD}_{3}$ & $\mathrm{FCP}_{1}$ & $\mathrm{FCP}_{2}$ & $\mathrm{FCP}_{3}$ \\
\hline $\begin{array}{l}\text { Bulk density } \\
\text { (gm/cc) }\end{array}$ & 0.57 & 0.50 & 0.49 & 0.51 & 0.50 & 0.51 & 0.52 & 0.50 & 0.54 & 0.55 & 0.52 & 0.53 & 0.54 \\
$\begin{array}{l}\text { Tapped density } \\
\text { (gm/cc) }\end{array}$ & 0.64 & 0.58 & 0.52 & 0.60 & 0.59 & 0.56 & 0.56 & 0.57 & 0.59 & 0.63 & 0.59 & 0.58 & 0.57 \\
Angle of repose ( ${ }^{\circ}$ ) & 30.10 & 32.10 & 28.10 & 32.52 & 29.68 & 31.52 & 29.68 & 31.02 & 30.90 & 32.50 & 31.18 & 32.14 & 33.04 \\
Carr's index (\%) & 10.14 & 10.11 & 8.11 & 10.10 & 9.12 & 10.11 & 8.92 & 10.52 & 10.19 & 9.14 & 11.00 & 7.76 & 7.05 \\
Hausner's ratio & 1.12 & 1.16 & 1.00 & 1.08 & 1.01 & 1.15 & 1.12 & 1.13 & 1.12 & 1.21 & 1.13 & 1.16 & 1.07 \\
\hline
\end{tabular}

Table 3. Post-compression parameters of formulations prepared by direct compression method.

\begin{tabular}{|c|c|c|c|c|c|c|c|c|c|c|c|c|c|}
\hline Parameters & $\mathrm{FC}_{0}$ & $\mathrm{FTS}_{1}$ & $\mathrm{FTS}_{2}$ & $\mathrm{FTS}_{3}$ & $\mathrm{FBA}_{1}$ & $\mathrm{FBA}_{2}$ & $\mathrm{FBA}_{3}$ & $\mathrm{FHD}_{1}$ & $\mathrm{FHD}_{2}$ & $\mathrm{FHD}_{3}$ & $\mathrm{FCP}_{1}$ & $\mathrm{FCP}_{2}$ & $\mathrm{FCP}_{3}$ \\
\hline $\begin{array}{l}\text { Hardness } \\
\left(\mathrm{Kg} / \mathrm{cm}^{2}\right)\end{array}$ & $\begin{array}{c}2.6 \\
\pm 0.070\end{array}$ & $\begin{array}{c}2.7 \\
\pm 0.070\end{array}$ & $\begin{array}{c}2.8 \\
\pm 0.099\end{array}$ & $\begin{array}{c}2.7 \\
\pm 0.070\end{array}$ & $\begin{array}{c}2.8 \\
\pm 0.099\end{array}$ & $\begin{array}{c}2.7 \\
\pm 0.070\end{array}$ & $\begin{array}{c}2.7 \\
\pm 0.070\end{array}$ & $\begin{array}{c}2.8 \\
\pm 0.099\end{array}$ & $\begin{array}{c}2.8 \\
\pm 0.099\end{array}$ & $\begin{array}{c}2.9 \\
\pm 0.070\end{array}$ & $\begin{array}{c}2.8 \\
\pm 0.099\end{array}$ & $\begin{array}{c}2.8 \\
\pm 0.099\end{array}$ & $\begin{array}{c}2.6 \\
\pm 0.070\end{array}$ \\
\hline $\begin{array}{l}\text { Thickness } \\
(\mathrm{mm})\end{array}$ & $\begin{array}{c}2.64 \\
\pm 0.020\end{array}$ & $\begin{array}{c}2.66 \\
\pm 0.020\end{array}$ & $\begin{array}{c}2.70 \\
\pm 0.010\end{array}$ & $\begin{array}{c}2.69 \\
\pm 0.010\end{array}$ & $\begin{array}{c}2.69 \\
\pm 0.010\end{array}$ & $\begin{array}{c}2.71 \\
\pm 0.020\end{array}$ & $\begin{array}{c}2.70 \\
\pm 0.070\end{array}$ & $\begin{array}{c}2.68 \\
\pm 0.020\end{array}$ & $\begin{array}{c}2.67 \\
\pm 0.010\end{array}$ & $\begin{array}{c}2.72 \\
\pm 0.020\end{array}$ & $\begin{array}{c}2.71 \\
\pm 0.010\end{array}$ & $\begin{array}{c}2.72 \\
\pm 0.080\end{array}$ & $\begin{array}{c}2.71 \\
\pm 0.010\end{array}$ \\
\hline $\begin{array}{l}\text { Friability } \\
(\%)\end{array}$ & $\begin{array}{c}0.62 \\
\pm 0.010\end{array}$ & $\begin{array}{c}0.58 \\
\pm 0.010\end{array}$ & $\begin{array}{c}0.60 \\
\pm 0.011\end{array}$ & $\begin{array}{c}0.59 \\
\pm 0.010\end{array}$ & $\begin{array}{c}0.61 \\
\pm 0.010\end{array}$ & $\begin{array}{c}0.62 \\
\pm 0.020\end{array}$ & $\begin{array}{c}0.59 \\
\pm 0.014\end{array}$ & $\begin{array}{c}0.58 \\
\pm 0.014\end{array}$ & $\begin{array}{c}0.59 \\
\pm 0.007\end{array}$ & $\begin{array}{c}0.60 \\
\pm 0.026\end{array}$ & $\begin{array}{c}0.61 \\
\pm 0.005\end{array}$ & $\begin{array}{c}0.60 \\
\pm 0.010\end{array}$ & $\begin{array}{c}0.59 \\
\pm 0.010\end{array}$ \\
\hline $\begin{array}{l}\text { In vitro } \\
\text { dispersion } \\
\text { time (sec) }\end{array}$ & $\begin{array}{c}110.23 \\
\pm 0.9\end{array}$ & $\begin{array}{c}67.64 \pm \\
1.01\end{array}$ & $\begin{array}{c}42.6 \\
\pm 1.990\end{array}$ & $\begin{array}{r}26.32 \\
\pm 1.01\end{array}$ & $\begin{array}{r}57.69 \\
\pm 0,01\end{array}$ & $\begin{array}{l}34.86 \\
\pm 0.58\end{array}$ & $\begin{array}{c}27.1 \\
\pm 1.005\end{array}$ & $\begin{array}{c}55.9 \\
\pm 0.995\end{array}$ & $\begin{array}{c}38.3 \\
\pm 1.006\end{array}$ & $25.02 \pm 0.01$ & $\begin{array}{c}60.2 \\
\pm 1.995\end{array}$ & $\begin{array}{l}23.70 \\
\pm 2.01\end{array}$ & $\begin{array}{l}16.40 \\
\pm 1.01\end{array}$ \\
\hline $\begin{array}{l}\text { Wetting } \\
\text { time (sec) }\end{array}$ & $\begin{array}{c}112.13 \\
\pm 1.0\end{array}$ & $\begin{array}{l}68.74 \\
\pm 1.97\end{array}$ & $\begin{array}{l}43.79 \\
\pm 0.97\end{array}$ & $\begin{array}{l}28.63 \\
\pm 0.97\end{array}$ & $\begin{array}{c}59.8 \\
\pm 1.012\end{array}$ & $\begin{array}{c}36.1 \\
\pm 1.040\end{array}$ & $\begin{array}{c}28.9 \\
\pm 0.980\end{array}$ & $\begin{array}{c}57.1 \\
\pm 0.980\end{array}$ & $\begin{array}{l}39.86 \\
\pm 0.01\end{array}$ & $\begin{array}{c}27.1 \\
\pm 2.010\end{array}$ & $\begin{array}{c}61.8 \\
\pm 1.005\end{array}$ & $\begin{array}{l}24.81 \\
\pm 0.99\end{array}$ & $\begin{array}{l}18.14 \\
\pm 0.97\end{array}$ \\
\hline $\begin{array}{l}\text { Water } \\
\text { absorption } \\
\text { ratio (\%) }\end{array}$ & $\begin{array}{r}56.20 \\
\pm 0.99\end{array}$ & $\begin{array}{c}68.1 \\
\pm 1.009\end{array}$ & $\begin{array}{l}74.55 \\
\pm 0.98\end{array}$ & $\begin{array}{l}81.87 \\
\pm 1.03\end{array}$ & $\begin{array}{c}72.1 \\
\pm 0.100\end{array}$ & $\begin{array}{c}78.1 \\
\pm 1.005\end{array}$ & $\begin{array}{c}83.5 \\
\pm 1.012\end{array}$ & $\begin{array}{c}72.0 \\
\pm 1.090\end{array}$ & $\begin{array}{c}76.4 \\
\pm 1.015\end{array}$ & $\begin{array}{c}84.3 \\
\pm 1.015 \mathrm{~s}\end{array}$ & $\begin{array}{c}71.0 \\
\pm 0.990\end{array}$ & $\begin{array}{l}84.44 \\
\pm 0.99\end{array}$ & $\begin{array}{c}95.4 \\
\pm 0.980\end{array}$ \\
\hline $\begin{array}{l}\text { Percent } \\
\text { drug } \\
\text { content } \\
\text { (\%) }\end{array}$ & $\begin{array}{c}97.8 \\
\pm 0.020\end{array}$ & $\begin{array}{c}97.8 \\
\pm 0.034\end{array}$ & $\begin{array}{c}97.9 \\
\pm 0.100\end{array}$ & $\begin{array}{c}97.9 \\
\pm 0.009\end{array}$ & $\begin{array}{l}98.16 \\
\pm 0.60\end{array}$ & $\begin{array}{c}98.2 \\
\pm 0.569\end{array}$ & $\begin{array}{c}98.1 \\
\pm 0.017\end{array}$ & $\begin{array}{l}98.28 \\
\pm 0.42\end{array}$ & $\begin{array}{c}98.2 \\
\pm 0.026\end{array}$ & $\begin{array}{c}98.1 \\
\pm 0.020\end{array}$ & $\begin{array}{c}97.8 \\
\pm 0.087\end{array}$ & $\begin{array}{c}97.9 \\
\pm 0.080\end{array}$ & $\begin{array}{l}98.47 \\
\pm 0.57\end{array}$ \\
\hline
\end{tabular}

Weight variation (148 -155 mg) within IP limits of $\pm 7.5 \%$, *Average of three determinations. 
Table 4. Comparative in-vitro dissolution parameters of promising fast dissolving tablet formulations, control and commercial conventional formulation (CCF) in ph 6.8 phosphate buffer.

\begin{tabular}{lccccccc}
\hline \multirow{2}{*}{$\begin{array}{l}\text { Formulation } \\
\text { code }\end{array}$} & \multicolumn{7}{c}{ Dissolution parameters } \\
\cline { 2 - 7 } & $\mathrm{D}_{5}(\%)$ & $\mathrm{D}_{10}(\%)$ & $\mathrm{D}_{15}(\%)$ & $\begin{array}{c}\mathrm{DE}_{10 \min } \\
(\%)\end{array}$ & $\mathrm{t}_{50 \%}(\mathrm{~min})$ & $\mathrm{t}_{70}(\mathrm{~min})$ & $\mathrm{t}_{90 \%}(\mathrm{~min})$ \\
\hline $\mathrm{FC}_{0}$ & $21 \%$ & $41 \%$ & $52 \%$ & $20.95 \%$ & $>30 \mathrm{~min}$ & $>30 \mathrm{~min}$ & $>30 \mathrm{~min}$ \\
$\mathrm{FTS}$ & $67 \%$ & $88.5 \%$ & $100.5 \%$ & $61.93 \%$ & $1.9 \mathrm{~min}$ & $4.9 \mathrm{~min}$ & $10 \mathrm{~min}$ \\
$\mathrm{FBA}_{3}$ & $53 \%$ & $84.10 \%$ & $92.5 \%$ & $59.07 \%$ & $2.1 \mathrm{~min}$ & $6.6 \mathrm{~min}$ & $13.8 \mathrm{~min}$ \\
$\mathrm{FHD}_{3}$ & $72.10 \%$ & $91.5 \%$ & $100.1 \%$ & $66.62 \%$ & $1.7 \mathrm{~min}$ & $4.7 \mathrm{~min}$ & $9.4 \mathrm{~min}$ \\
$\mathrm{FCP}_{3}$ & $79.5 \%$ & $100 \%$ & - & $71.82 \%$ & $1.5 \mathrm{~min}$ & $2.9 \mathrm{~min}$ & $7.1 \mathrm{~min}$ \\
$\mathrm{CCF}$ & $21 \%$ & $46 \%$ & $52.5 \%$ & $22.99 \%$ & $13 \mathrm{~min}$ & $2.9 \mathrm{~min}$ & $>30 \mathrm{~min}$ \\
\hline
\end{tabular}

Table 5. Stability data of $\mathrm{FHD}_{3}$ formulation at $40^{\circ} \mathrm{C} / 75 \% \mathrm{RH}$.

\begin{tabular}{ccccc}
\hline $\begin{array}{c}\text { Sl. } \\
\text { No. }\end{array}$ & Time in days & Physical changes & $\begin{array}{c}\text { Percent drug content } \\
\pm \text { SD }^{*}\end{array}$ & $\begin{array}{c}\text { In-vitro dispersion } \\
\text { time* }\end{array}$ \\
\hline 1. & $1^{\text {st }}$ day (initial) & -- & $98.10 \pm 0.0346$ & $25.02 \pm 0.019$ \\
2. & $30^{\text {th }}$ day (1 month) & No changes & $98.28 \pm 0.0699$ & $25.24 \pm 0.142$ \\
3. & $60^{\text {th }}$ day (2 month) & No changes & $98.36 \pm 0.0721$ & $25.51 \pm 0.026$ \\
4. & $90^{\text {th }}$ day (3 month) & No changes & $98.40 \pm 0.173$ & $25.72 \pm 0.017$ \\
\hline
\end{tabular}

* Average of three determinations

Table 6. Statistical analysis for drug content data of $\mathrm{FHD}_{3}$ formulation.

\begin{tabular}{ccccc}
\hline $\begin{array}{c}\text { Sl. } \\
\text { No. }\end{array}$ & Trials & $1^{\text {st }}$ day $(\mathrm{A})$ & $90^{\text {th }}$ day $(\mathrm{B})$ & $\mathrm{A}-\mathrm{B}$ \\
\hline 1. & 1 & 97.75 & 97.60 & 0.15 \\
2. & 2 & 98.35 & 98.10 & 0.25 \\
3. & 3 & 98.20 & 98.01 & 0.19 \\
4. & Mean \% drug content & 98.10 & 97.90 & 0.20 \\
5. & \pm SD & 0.3122 & 0.266 & 0.046 \\
\hline $\mathrm{t}=2.50$ & $(\mathrm{p}<0.05)$ & & &
\end{tabular}

Table 7. Kinetic data of promising fast dissolving tablet formulations, control and conventional commercial formulation (CCF) in ph 6.8 buffer.

\begin{tabular}{lccc}
\hline \multicolumn{2}{l}{ Formulation code } & Zero-order & First-order \\
\hline \multirow{2}{*}{$\mathrm{FC}_{0}$} & $\mathrm{R}$ & 0.7886 & 0.954 \\
& A & 28.075 & 1.956 \\
& $\mathrm{~B}$ & 8.3993 & 0.0145 \\
$\mathrm{FTS}_{3}$ & $\mathrm{R}$ & 0.6753 & 0.9831 \\
& $\mathrm{~A}$ & 35.81 & 1.9582 \\
& $\mathrm{~B}$ & 5.474 & 0.018 \\
$\mathrm{FBA}_{3}$ & $\mathrm{r}$ & 0.7162 & 0.9902 \\
& A & 32.908 & 1.8662 \\
& $\mathrm{~B}$ & 5.5349 & 0.0661 \\
$\mathrm{FHD}_{3}$ & $\mathrm{r}$ & 0.7689 & 0.6828 \\
& $\mathrm{~A}$ & 29.28 & 1.6514 \\
& $\mathrm{~B}$ & 5.6382 & 0.0675 \\
$\mathrm{FCP}_{3}$ & $\mathrm{r}$ & 0.9217 & 0.9634 \\
& A & 11.969 & 1.8386 \\
& B & 2.287 & 0.1016 \\
$\mathrm{CCF}_{3}$ & $\mathrm{r}$ & 0.9075 & 0.9633 \\
& A & 10.544 & 1.7795 \\
& B & 2.0564 & 0.1085 \\
\hline
\end{tabular}




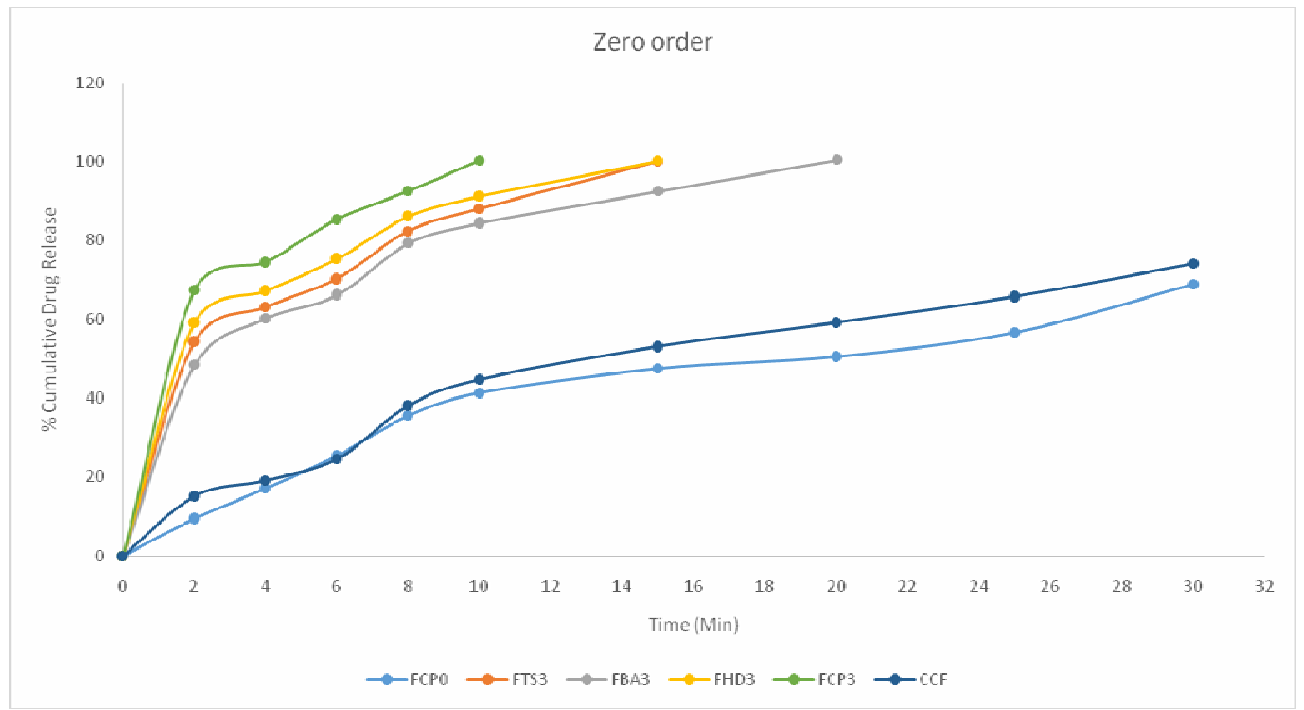

Figure 1. Comparative cumulative \% drug release versus time plots (zero-order) of promising fast dissolving tablet formulations, control and conventional commercial formulations (CCF) in ph 6.8 phosphate

ratio of $\mathrm{FHD}_{3}$ were found to be $25.02 \mathrm{sec}, 27.12 \mathrm{sec}$ and $84.35 \%$ respectively (Table 3 ). The experimental data also revealed that the results obtained from the H. sabdariffa mucilage are better than those of conventional commercial formulation.

In vitro dissolution studies of the control formulation $\left(\mathrm{FC}_{0}\right)$, commercial conventional formulation (CCF) and promising formulations $\left(\mathrm{FHD}_{3}, \mathrm{FCP}_{3}\right)$ were carried out at $\mathrm{pH} 6.8$ phosphate buffer and the various dissolution parameters, such as percent drug dissolved in $5 \mathrm{~min}, 10 \mathrm{~min}$ and $15 \mathrm{~min}$ $\left(D_{5}, D_{10}\right.$ and $\left.D_{15}\right)$, dissolution efficiency at $10 \mathrm{~min}$ $\left(\mathrm{DE}_{10}\right), \mathrm{t}_{50 \%}, \mathrm{t}_{70 \%}$ and $\mathrm{t}_{90 \%}$ as shown in table 4 and dissolution profile as depicted in figure 1 . These data reveal that overall formulation $\mathrm{FHD}_{3}$, which showed more than eight fold faster drug release $\left(t_{50 \%} \quad 1.7\right.$ min) when compared to CCF ( $\mathrm{t}_{50 \%} 13.0 \mathrm{~min}$ ) tablet of nebivolol and released nearly more than 18 fold than control formulation.

IR spectroscopic studies indicated that the drug is compatible with all the excipients. The IR spectrum of $\mathrm{FHD}_{3}$ and $\mathrm{FCP}_{3}$ showed all the characteristics peaks of nebivolol pure drug, thus conforming that no interaction of drug occurred with the component of the formulations. Stability studies of the $\mathrm{FHD}_{3}$ formulation presented in table 5 and table 6 indicated that there is no significant changes in drug content and in vitro dispersion time at the end of three months period $(\mathrm{p}<0.05)$.

\section{CONCLUSION}

From the present work, it can be concluded that fast dissolving tablets of nebivolol prepared by using mucilage of $H$. sabdariffa showed better drug release and disintegration time as compared to tablets prepared from other natural and synthetic disintegrants.

\section{ACKNOWLEDGEMENT}

The authors are grateful to the management and staff of H.K.E. Society's College of Pharmacy, Gulbarga, Karnataka for providing facilities to carry out the work.

\section{REFERENCES}

1. Government of India, Ministry of Health \& Family Welfare 2010. Indian Pharmacopoeia Delhi Controller of Publications, p. 849.

2. Seager, H. 1998, Drug delivery products and the zydis fast dissolving dosage forms. J. Pharm.Pharmacol. 50, 378-382

3. Chang, R.K., Guo, X., Burnside, B.A. and Cough, R.A. 2000. Fast dissolving tablets. Pharm. Tech. 24, 52-58. 
4. Dobetti, L. 2001. Fast-melting tablets: Developments and Technologies. Pharm. Tech. pp. 44-50.

5. Kuchekar, B.S. and Arumugam, V. Fast Dissolving Tablets. Indian. J. Pharm Edu. 20, 35150-351502.

6. Baveja, S.K. and Gupta, B.M. 1968. Rheology of Aqueous dispersions of Plantago ovata seed husk-I. Ind. J. Pharm. Sci. 30, 187-94.

7. Baveja, S.K. and Gupta, B.M. 1968. Rheology of Aqueous dispersions of Plantago ovata seed husk-II. Ind. J. Pharm. Sci. 30, 247-251

8. Mithal, B.M. and Kasid, J.L. 1964. Evaluation of emulsifying properties of Plantago ovata (Ispaghula) seed husk. Ind. J. Pharm. Sci. 26, 316-319.

9. Mithal, B.M. and Kasid, J.L. 1965. Evaluation of the suspending properties of Plantago ovata (Ispaghula) seed husk. Ind. J. Pharm. Sci. 27, 331-335.

10. Kulkarni, G.T., Gowthamarajan, K., Rao, B.G. and Suresh, B. 2002. Evaluation of binding property of Plantago ovata and Trigonella Foenum gracecum mucilage. Ind. Drugs. 39, 422425.

11. Washi, S.P., Sharma, V.D., Jain, V.K. and Sinha, P. 1985. Plantago ovata: genetic diversity, cultivation, utilization and chemistry. Ind. J. Nat. Prod. 1, 3-6.
12. Rupa, S. and Banik, J.K. 2011. Evaluation of Hibiscus sabdariffa leaf mucilage. Inter. J. Pharm. Pharm.Sci. 3, 184187.

13. Kuchekar, B.S., Badhan, A.C. and Mahajan, H.S. 2004. Mouth dissolving tablets of salbutamol sulphate: A novel drug delivery system. Ind. Drugs. 41, 592-598.

14. Liberman, H. and Lachman, L. 1991. The Theory and Practice of Industrial Pharmacy, $3^{\text {rd }}$ ed. Bombay. Verghese Publication House. 2, 171-193.

15. Jeevanandham, S., Dhachinamoorthi, D., Chandrashekhar, K.B., Muthukumaran, M. and Sriram, N. 2010. Formulation and evaluation of naproxen sodium orodispersible tablets- A sublimation technique. Asian . J. Pharm. 4, 48-51

16 Chaudhari, P.D., Chaudhari, S.P., Kolhe, S.R., Dave, K.V., More, D.M. 2005. Formulation and Evaluation of fast dissolving tablets of famotidine. Indian. Drugs . 42, 641-649.

17. Bi, Y.X., Sunada, H., Yonezawa, Y., Danjo, K. 1999. Evaluation of rapidly disintegrating tablets by direct compression method. Drug. Dev. Ind. Pharm. 25, 571-581.

18. Bhagwati, S.T., Hiremath, S.N., Sreenivas, S.A. 2005. Comparative evaluation of disintegrants by formulation cefixime dispersible tablets. Indian. .J Pharm. Edu. Res. 39, 194-197. 